\title{
A Novel Point of Care Diagnostic Device: Impedimetric Detection of a Biomarker in Whole Blood
}

\author{
Amanda A McMurray, Zulfiqur Ali, Jan Kyselovik, Christopher A. Mills, Nicole Jaffrezic Renault, \\ Hunor Santha, Christof Strohhöfer
}

\begin{abstract}
There is an unmet medical need for a more reliable and earlier assessment of patients suspected of having a deep vein thrombosis.

We describe a novel approach which is developing a highly reliable, accurate, portable and handheld prototype medical diagnostic device to improve radically the speed, accuracy and reliability with which DVT and related blood clotting conditions can be assessed. The device will measure whole blood concentration of D-dimer, a recognized biomarker of increased blood clotting activity, and through innovation in the development of a novel detection, measurement and reporting system, will offer the opportunity to use the test in the point of care setting.
\end{abstract}

The device combines innovation in antibody bio-engineering for high specificity immunoassay-based diagnostics and nano/micro engineered impedimetric analysis electrodes incorporating a biocompatible polymer substrate with development of a disposable microfluidic manifold specifically enabling diagnostics at the point-of-first-contact.

\section{INTRODUCTION}

\section{$\mathrm{D}$} IAGNOSIS and hence treatment of disease is often reliant on the interpretation of chemical parameters within biological samples. Currently many of these measurements

Manuscript received April 2, 2007.

This work was supported in part by the European Commission under Framework Programme 6 (Deep Vein Thrombosis Impedimetric Microanalysis System "DVT-IMP", contract number 034256)

Amanda McMurray is with Cenamps, The Centre of Excellence in Nano and Micro Photonic Systems, Fabriam Centre, Newcastle upon Tyne, NE28 9NZ, UK (phone: +44 191280 4273; fax: +44 191280 5525; email: amanda.mcmurray@cenamps.com).

Nicole Jaffrezic Renault is with the Laboratory of Analytical Chemistry, Claude Bernard University Lyon 1, 69622 Villeurbanne cedex, France (email: Nicole.Jaffrezic@univ-lyon1.fr).

Jan Kyselovik is with Comenius University, Bratislava Solvakia (email: kyselovik@fpharm.uniba.sk

Christopher A. Mills is with the Nanobioengineering Research Laboratory, Bioengineering Institute of Catalonia (IBEC), Barcelona Science Park, C/ 1-5, Barcelona, Spain. (e-mail: cmills@pcb.ub.es).

Hunor Santha is with the Budapest University of Technology and Economics, Mûegyetem rkp. 3-9, H-1111 Budapest, Hungary (email: santha@ett.bme.hu)

Christof Strohhöfer is with the Fraunhofer-Gesellschaft zur Förderung der angewandten Forschung e.V., Postfach 2007 33, 80007 München,

Germany (e-mail: christof.strohhoefer@izm-m.fraunhofer.de).

Zulfiqur Ali is in the School of Science and Technology, University of Teesside, Middlesbrough, UK (email: z.ali@tees.ac.uk) are performed in centralized laboratories, requiring specialized equipment and expert personnel. This approach, whilst delivering high quality results, can delay diagnosis and hence delay commencement of treatment. There is an emerging requirement for diagnosis to be made at point of first contact with the patient, requiring clinical measurements being made outside the laboratory using compact, portable devices that can be operated by nonspecialised people. This approach, known as point-of-care testing, is particularly applicable when there is a need for rapid diagnosis and fast initiation of treatment.

Venous thromboembolism represents a single disease entity with two patterns of clinical presentation: deep vein thrombosis and pulmonary embolism. Deep vein thrombosis (DVT) is an internal clot formed in one of the body's deep veins, usually one of those in the leg. If a part of the clot breaks free and moves into the lung, it can lead to pulmonary embolism (PE), an often-fatal condition. Venous thromboembolism afflicts an estimated 71 per 100,000 persons yearly, and DVT and PE are major causes of unexpected mortality in hospitals throughout Europe [1-4]. It is essential that diagnosis is made quickly and accurately and that treatment is started promptly.

During 2005 the number of reported incident cases of DVT and PE totalled 1.3 million in France, Spain, Germany, Italy, United Kingdom, Japan and United States alone. This figure is expected to rise to 1.4 million per annum by 2011 , due to increase in population. Within these countries it is estimated that healthcare systems invest up to $€ 6.6$ billion annually in assessment of patients suspected to be suffering from thromboembolitic event, whose diagnosis proves to be negative.

Clinical practice guidelines [5-7] indicate that the accuracy of diagnosis of DVT/PE by measurement of Ddimer concentration in combination with radiological or laboratory diagnostic tests is superior to traditional methods of diagnosis [8,9]. Hence, D-dimer, a recognised biomarker for the diagnosis of a thrombus, is routinely measured in hospital laboratories using a traditional ELISA technique. Ddimer tests are generally highly sensitive but not highly specific, suggesting that negative tests are much more useful for ruling out DVT than are positive tests for ruling in DVT [10-13]. The negative predictive value (proportion of 
patients with a negative test who do not have DVT) for patients with a negative D-dimer blood test and a low clinical probability of DVT is higher than $99 \%$ [13-15].

However since most quantitative D-dimer tests are performed in the laboratory, these can generally only be offered in a hospital clinic hence patients cannot be fully assessed within primary care: they are referred for assessment to a secondary care setting, such as a clinic or hospital, where a combination of laboratory based blood test and expert clinical assessment is performed. This causes potentially life-threatening delays to diagnosis and commencement of treatment for patients who do indeed have a blood clot; it also causes unnecessary delays for patients who do not.

An analysis of current medical diagnostic practice and available tests indicates that practitioners in primary care, such as GP surgeries and community clinics would welcome access to a reliable point of care D-dimer test as it would improve the quality of care that they can provide to their patients, improve DVT healthcare diagnosis and treatment services, and hence improve patient outcomes.

Development of an innovative medical diagnostic device to measure whole blood D-dimer concentration at point of care is described. It comprises a novel immunochemical biosensor and detection system within a disposable cartridge and with a reader for data management and transfer.

The development of a thin film biosensor, comprising an antibody captured onto the surface of a printed electrode and capable of measuring concentration of a biomarker in whole blood, is described and the development of impedimetric detection, with appropriate blood handling, for measurement of biomarkers in whole blood is discussed. Concentration of D-dimer antigen in a sample of whole blood is measured by a novel impedimetric measurement system. The biosensor and detection system lie within a disposable cartridge, containing innovative whole blood handling capabilities and test and data management and transfer system are held within a base unit.

Finally, the development of a disposable liquid handling cartridge, with microfluidic system designed to manipulate a whole blood sample and capable of delivering the sample, in appropriate form, into the impedimetric measurement system, is described.

\section{DEVELOPMENT OF CLINICAL RECOMMENDATIONS FOR ASSESSMENT OF DVT AND PE}

The clinical goals addressed are as follows:

(1) Are clinical prediction rules valuable for diagnosing DVT/PE, and how does addition of our novel D-dimer assay best improve the test characteristics of clinical prediction rules?

(2) What are the test characteristics of D-dimer measurement alone when used for diagnosis or exclusion of DVT or PE, and how does choice of assay affect the test characteristics?

(3) What are the test characteristics of D-dimer vs. ultrasonography and or computed axial tomography?

Evaluation of the performance of the experimental model assay, by comparison with the known clinical status of the blood samples and comparison with the performance of existing D-dimer testing regimes, is described.

\section{DETECTION OF A BIOMARKER IN WHOLE BLOOD USING A THIN FILM BIOSENSOR WITH IMPEDIMETRIC DETECTION}

Whole blood D-dimer concentration is measured through detection of change in impedance at the measurement electrode as a result of binding of D-dimer molecules to antibodies that have been bioengineered and immobilized onto the electrode surface, thus creating a highly specific immunoassay.

\section{A. Development of the Biosensor}

Biosensors increasingly use thin films incorporating active organic molecules. These layers either interface with, or act as, the transduction element of the sensor. The immobilisation of the active molecules can take a number of forms; for example, as monolayers directly attached to the sensor, as components in thin polymer films, or immobilised on supports, such as nanoparticles. In electrochemical biosensors, the active layer is interfaced with electrodes which react to changes in the electronic structure of the active layer, and which are detected by the measurement electronics. These changes, which are produced by chemical interactions between the molecules in the active layer and the analyte of interest, can be detected using a number of measurement techniques [16].

Commonly, microelectrodes produced from suitable conducting materials, such as gold, platinum, and silver are used as the interface electrodes. However, the development of techniques such as nanoimprint lithography (NIL) [17], focused ion beam (FIB) milling [18] and laser ablation, complemented by high-resolution etching techniques such as reactive ion etching (RIE) [19], have lead to the production of nanoelectrodes [20]. These nanoelectrode geometries potentially have advantages with respect to their microfabricated analogues because they have improved spatial resolution and reduced signal noise [21].

Interfacing of the active layer molecules to nanoelectrodes is not a trivial subject, and we describe our evaluation and selection of appropriate technique. For example, if the molecules can be incorporated into a monomer precursor, they can be immobilised onto the electrodes using 
electropolymerisation [22]. In this case, the polymer itself may need to be conducting to facilitate electron transfer to the electrode surface [23]. Common electrically conducting polymers used in sensing applications include poly(pyrrole) [24] and poly(aniline) [25] and their derivatives. The active (bio)molecules can be covalently bonded to the polymers, using suitable coupling reactions, which may involve the use of reactive moieties, such as amine, acid or thiol groups, or simply immobilised in the polymer matrix. Positional control of the active layer using this technique is facile, as the polymer grows predominantly on the electrode surface. However, the addition of the supporting polymer in the sensing system introduces a level of complexity when modelling the sensors and when attempting to produce a number of sensors with high reproducibility.

Direct immobilisation of the active (bio)molecules to the surface of an electrode can be completed using covalent bonding. One of the most commonly used techniques for this is the interaction between a thiol group and a gold electrode. For example, this technique has been extensively used for the immobilisation of (bio)molecules through self assembly [26]. Such self-assembly can be used to immobilise biological material [27] and can be positionally controlled using a soft lithography method, called micro-contact printing [28], down to nanometric scales [29]. The formation of a thiol linkage between the gold and the thiolated (bio)molecule means that the active molecule is directly linked to the electrode. Non-specific immobilisation either side of the electrodes should be avoided as long as the thiol group has no affinity for the underlying substrate. The same molecules can be deposited using dip-pen lithography [30].

We have produced nanoelectrodes on polymeric substrates and describe immobilization of active (bio)molecules using electropolymerisation, micro-contact printing or dip-pen lithography techniques, ready for incorporation of functionalised electrodes into our microfluidic cartridge system for the detection of D-dimer in whole blood at levels of $<300 \mathrm{ng} / \mathrm{ml}$. A variety of data extraction approaches are available including the measurement of impedance across a number of frequencies [31].

\section{B. Electrochemical Impedance Spectroscopy (EIS) label- free detection}

To achieve a sensitivity of $1 \mathrm{ng} / \mathrm{ml}$ in a range of 0 $3000 \mathrm{ng} / \mathrm{ml}$ of D-dimer in whole blood there is a need to measure impedance change on the electrode with high precision.

Electrochemical Impedance Spectroscopy (EIS) is a rapidly developing electrochemical technique for the characterization of biomaterial-functionalized electrodes and especially for the transduction of biosensing events at the electrodes. Experiments have indicated that antibody loaded electroconductive matrices (particularly polypyrrole) give concentration dependant responses when interrogated with
EIS [32,33]. The electroconductive matrix presents a high surface concentration of grafted biomolecules and the change of conformation brought by biological recognition induces a change of the polymer conjugation, which results in amplification of the electrical phenomenon. A particular enhancement of the sensitivity of detection was observed in presence of redox probe [34]. EIS, under appropriate experimental conditions, represents a label-free technique for immunodetection.

We have developed an approach to optimize the orientation control of the reduced antibody-scFV fragments in the electroconductive matrix. Further, we control the electronic exchange by inclusion of a specific redox species in the polymer structure, in order to enhance the sensitivity of detection and limit non specific adsorption onto the functionalised electrode surface, by grafting a specific group onto the pyrrole monomer.

\section{DEVELOPMENT OF A NOVEL MICROFLUIDIC SYSTEM, DEVICE AND READER}

\section{A. The Disposable Cartridge and liquid handling system}

We have designed the cartridge to ensure robustness of sample and reagent transfer and, therefore, focus on fluidic transfer properties rather than additional functionality.

The cartridge is designed and fabricated using established technologies in polymer microfabrication. These include hot embossing to define the microfluidic channels and chambers, photolithography and nanoimprint lithography to structure the impedimetric electrodes and laser ablation for the passivation layer.

Our design and choice of mass manufacturing approach takes into account consideration of future scalability.

The nano- and microelectrodes for the impedimetric measurement are housed in a sample chamber that can be accessed from both sample and reagent reservoirs.

Immobilisation of the D-dimer antibody onto the electrode is performed in an open cartridge configuration. This facilitates the immobilisation process and allows easy quality control of the biological receptor layers. However, it puts very high demand on the joining of the cartridge parts. We describe our choice of existing and novel joining processes that minimize temperature, UV and solvent load to the biological receptor molecules while guaranteeing robust functionality of the cartridge.

\section{B. Reader - Design and Functionality}

During the measurement phase 'cartridge' and 'reader' will operate as one compound system to provide all required functionalities. We describe, therefore, our design for the device as a whole, which has been conceptualized and developed to be user friendly, and able to be operated by 
non-expert users, such as practitioners, paramedics and ultimately by patients.

The reader itself is a microcontroller driven device, part of a complex system consisting of four component parts that, when combined, give rise to a device capable of measuring biomarker concentration under Point-of-Care conditions.

We have developed an approach to drive the blood sample from point of entry to the electrode and then to remove the unwanted blood sample components (plasma, red blood cells, other proteins, etc.) from the measurement site. It relies on fluidic interconnection between cartridge and reader and on a micro-pump situated in the reader that will move buffer solution and blood sample through the cartridge.

\section{ACKNOWLEDGMENT}

The Authors thank the DVT-IMP Project team www.diagnsosingdvt.com, a Consortium of ten leading European research groups and high-tech companies, led by University of Teesside, Helena Bioscience Ltd and Cenamps, for their valuable feedback to this document.

\section{REFERENCES}

[1] Heit JA, Melton LJ 3rd, Lohse CM, et al. Incidence of venous thromboembolism in hospitalized patients vs community residents. Mayo Clin Proc. 2001;76(11):1102-1110.

[2] White RH. The epidemiology of venous thromboembolism. Circulation. 2003;107(23 Suppl 1):I4-8.

[3] Fowkes FJ, Price JF, Fowkes FG. Incidence of diagnosed deep vein thrombosis in the general population: systematic review. Eur J Vasc Endovasc Surg. 2003(1);25:1-5.

[4] Anderson FA, Jr., Wheeler HB, Goldberg RJ, et al. A populationbased perspective of the hospital incidence and case-fatality rates of deep vein thrombosis and pulmonary embolism. The Worcester DVT Study. Arch Intern Med. 1991;151(5):933-938.

[5] Segal JB, Eng J, Jenckes MW, et al. Diagnosis and Treatment of Deep Venous Thrombosis and Pulmonary Embolism. Evidence Report/Technology Assessment No. 68. Rockville, Md: Agency for Healthcare Research and Quality. March 2003. AHRQ publication 03E016.

[6] Finnish Medical Society Duodecim. Deep venous thrombosis. Helsinki, Finland: Duodecim Medical Publications Ltd; 2002. Available at: http://www.ebm-guidelines.com.

[7] American College of Emergency Physicians-Medical Specialty Society. Clinical policy: critical issues in the evaluation and management of adult patients presenting with suspected lowerextremity deep venous thrombosis. Ann Emerg Med. 2003;42:124135

[8] Wells PS, Anderson DR, Bormanis J, et al. Value of assessment of pretest probability of deep-vein thrombosis in clinical management. Lancet. 1997;350:1795-1798.

[9] Wells PS, Hirsh J, Anderson DR, et al. A simple clinical model for the diagnosis of deep vein thrombosis combined with impedance plethysmography: potential for an improvement in the diagnostic process. J Intern Med. 1998;243:15-23.

[10] Kelly J, Rudd A, Lewis RR, Hunt BJ. Plasma D-dimers in the diagnosis of venous thromboembolism. Arch Intern Med. 2002;162: 747-756.

[11] Hansson PO, Eriksson H, Eriksson E, et al. Can laboratory testing improve screening strategies for deep vein thrombosis at an emergency unit? J Intern Med. 1994;235:143-151.
[12] Kearon C. Ginsberg JS, Douketis J, et al. Management of suspected deep vein thrombosis in outpatients by using clinical assessment and D-dimer testing. Ann Intern Med. 2001;135:108-111.

[13] Kearon C, Ginsberg JS, Douketis J, et al. Diagnosis of a first DVT in outpatients: interim analysis of a management study based on clinical evaluation and D-dimer results. Thromb Haemost. 1997;78:588.

[14] Wells PS, Anderson DR, Ginsberg J. Assessment of deep vein thrombosis or pulmonary embolism by the combined use of clinical model and noninvasive diagnostic tests. Semin Thromb Hemost. 2000;26: 643-656.

[15] Michiels JJ, Freyburger G, Van Der Graaf F, et al. Strategies for the safe and effective exclusion and diagnosis of deep vein thrombosis by the sequential use of clinical score, D-dimer testing and compression ultrasonography. Semin Thromb Hemost. 26, 657-667, 2000.

[16] Gerard, M., Chaubey, A., Malhotra, B. D., Biosens. Bioelectron. 17, 345-359, 2002

[17] S. Y. Chou, P. R. Krauss, P. J. Renstrom, Appl. Phys. Lett. 67, 31143116, 1995; S. Y. Chou, P. R. Krauss, P. J. Renstrom, Science 272, 85-87, 1996.

[18] Tseng A. A., J. Micromech. Microeng. 14, R15-R34, 2004.

[19] Lang W., Mater. Sci. Eng. R: Reports, 17, 1-55, 1996.

[20] Gerwen, P. V., Laureyn, W., Laureys, W., Huyberechts, G., De Beeck, M. O., Baert, K., Suls, J., Sansen, W., Jacobs, P., Hermans, L., Mertens, R., Sens. Act. B: Chem. 49, 73-80, 1998.

[21] Kleps I., Angelescu A., Miu M., Mater. Sci. Eng. C., 19, 219-223, 2002; Moretto L. M., Pepe N., Ugo P., Talanta 62, 1055-1060, 2004.

[22] Cosnier S., Biosens. Bioelectron., 14, 443-456, 1999.

[23] Saxena V., Malhotra B. D., Curr. Appl. Phys., 3, 293-305, 2003.

[24] Geetha S., Raoa C. R. K., Vijayana M., Trivedi D. C., Anal. Chim. Acta, 568, 119-125, 2006; Ramanavičius A., Ramanavičienè A., Malinauskas A., Electrochim. Acta. 51, 6025-6037, 2006.

[25] Zhang D., Wang Y., Mater. Sci. Eng. B., 134, 9-19, 2006.

[26] Love, J. C., Estroff, L. A., Kriebel, J. K., Nuzzo, R. G., Whitesides, G. M., Chem. Rev. 105, 1103-1169, 2005; Martelet C., Jaffrezic-Renault N., Hou Y., Errachid A., Bessueille F., "Self Assembled Monolayers for biosensing", Biosensors: Electrochemical And Mechanical Biosensors (Methods Express) (ed. Paul Millner) Scion Publishing UK, ISBN: 1904842127.

[27] Hou Y., Helali S., Zhang A., Jaffrezic-Renault N., Martelet C., Minic J., Gorojankina T., Persuy M-A., Pajot-Augy E., Salesse R., Bessueille F., Samitier J., Errachid A., Akinov V., Reggiani L., Pennetta C., Alfinito E., Biosens. Bioelectron. 21, 1393-1402, 2006.

[28] Xia Y., Whitesides G. M., Angew. Chem. Int. Ed. 37, 550-575, 1998.

[29] Renault J. P., Bernard, A., Bietsch, A., Michel, B., Bosshard, H. R., Delamarche, E., Kreiter, M., Hecht, B., Wild, U. P., J. Phys. Chem. B., 107, 703-711, 2003; Hong-Wei L., Beinn, V.O., Muir, G. F., Huck, W. T. S., Langmuir, 19, 1963-65, 2003.

[30] Piner, R. D., Zhu, J., Xu, F., Hong, S., Mirkin C. A., Science 283, 661-663, 1999.

[31] Pejcica B., De Marco R., Electrochim. Acta, 51, 6217-6229, 2006; Minic J., Grosclaude J., Aioun J., Persuy M-A., Gorojankina T., Salesse R., Pajot-Augy E., Helali S., Hou Y., Jaffrezic-Renault N., Bessueille F., Errachid A., Gomila G., Ruiz O., Samitier J., Biochim. Biophys. Acta. 1724, 324-332, 2005.

[32] O. Ouerghi, A. Touhami, N. Jaffrezic-Renault, C. Martelet, H. Ben Ouada, S. Cosnier "Electrodeposited biotinylated polypyrrole as immobilization method for impedimetric immunosensors" IEEE Sensors Journal, 4(5) (2004) 559-567

[33] C. Tlili, Hafsa Korri-Youssoufi, Laurence Ponsonnet, Claude Martelet, N. J. Jaffrezic-Renault "Electrochemical impedance probing of DNA hybridisation on oligonucleotide-functionalised polypyrrole" Talanta 68 (2005) 131-137

[34] S. Ameur, C. Martelet, N. Jaffrezic-Renault, J.M. Chovelon, "Sensitive immunodetection through impedance measurements onto gold functionalized electrodes" Applied Biochemistry and Biotechnology, 89(2-3) (2000) 161-170 